\title{
Glucose and Lipid Metabolism in Spontaneously Diabetic Torii Rat
}

\author{
Yasuko Mera, Hisayo Morinaga, Takeshi Ohta and Tomohiko Sasase* \\ Japan Tobacco Inc., Central Pharmaceutical Research Institute, Biological/Pharmacological Research Laboratories, \\ 1-1 Murasaki-cho, Takatsuki, Osaka 569-1125, Japan
}

\begin{abstract}
The impairment of glucose metabolism may lead to type 2 diabetes and the malfunction of lipid metabolism contributing to metabolic syndrome, which increases risk of heart disease, vascular disease, and type 2 diabetes. Our previous study provided evidence for involvement of hepatic glucose metabolic disorder in onset and progress of diabetes in Spontaneously Diabetic Torii (SDT) rats. As it is increasingly important to elucidate the mechanism of onset of diabetes and to develop drugs to prevent diabetic pathological progress, SDT rat should offer a highly useful model of spontaneous diabetic onset for such studies. In addition, abnormality in triglyceride (TG) absorption and impaired lipid catabolism antecedent to hypoinsulinemia/hyperglycemia seem to cause postprandial hypertriglyceridemia in SDT rat; hence, the characteristics of lipid metabolism in SDT rat can be useful in studies of diabetic hypertriglyceridemia and TG metabolism.
\end{abstract}

Keywords: SDT rat, hyperglycemia, hypertriglyceridemia, liver, intestine.

\section{INTRODUCTION}

In recent years, great attention has been focused on high risks of development of cardiovascular diseases in non-diabetic patients with glucose metabolic disorder and of diabetes in patients with glucose metabolic disorder. Similarly, hypertriglyceridemia is one of the major abnormalities found in diabetes with insulin deficiency [1], and is thought closely related to coronary heart disease and atherosclerosis, important complications of diabetes [2, 3]. Failures in any of the metabolic processes can lead to serious health problems: the impairment of glucose metabolism may lead type 2 diabetes, and the malfunction of lipid metabolism contributes to metabolic syndrome, which is a cluster of conditions that puts people at increased risk of heart disease, vascular disease, and type 2 diabetes. These epidemiological situations make it important to study diabetic pathogenesis to prevent progress to diabetes.

Among studies on the developmental stage of diabetes, particularly little has been published on hepatic glucose metabolism and triglyceride (TG) metabolism. In our previous studies, we focused on the liver, the major organ involved in glucose metabolism, to investigate changes in hepatic glucose metabolism from pre-diabetic to diabetic stages, with the aim of identifying the molecules involved in onset of diabetes [4]. Furthermore, we examined the contribution of TG absorption and clearance to diabetic hypertriglyceridemia in Spontaneously Diabetic Torii (SDT) rats [5]. SDT rat is a model of non-obese type 2 diabetes with impaired secretion of insulin [6,7]. In male SDT rats, plasma glucose levels spontaneously increased around 16 weeks of age (Fig. 1A). The plasma insulin levels of SDT rats at 20 weeks or older age were significantly lower than

*Address correspondence to this author at the Japan Tobacco Inc., Central Pharmaceutical Research Institute, Biological/Pharmacological Research Laboratories, 1-1 Murasaki-cho, Takatsuki, Osaka 569-1125, Japan; Tel: +81 72681 9700; Fax: +81 72681 9722; E-mail: tomohiko.sasase@jt.com those of age-matched Sprague-Dawley (SD) rats (Fig. 1B). Significant decreases in body weight were observed in diabetic SDT rats. The plasma TG and total cholesterol levels remained unchanged at 16 weeks of age, but plasma TG level increased after 30 weeks of age (Fig. 1C). Interestingly, increased plasma TG level elevation after oil loading was observed in SDT rats before hyperglycemia/ hypertriglyceri-demia [5], as was impaired glucose tolerance in pre-diabetic state [8]. Most of all, the characteristic that onset of diabetes is delayed until around 20 weeks of age makes this animal a highly effective model in which to pursue the mechanism of development of diabetes.

\section{GLUCOSE METABOLISM}

Glucokinase (GK) plays a critical role in metabolism of glucose in liver because it appears at the first step of glycolysis [9]. In SDT rat, decreased mRNA expression and activity of GK were observed from pre-diabetic 16 weeks of age [4], suggesting that a decrease in GK is partly responsible for a mild increase in blood glucose in SDT rat. Suppressed GK is found in NIDDM patients $[10,11]$ and some diabetic animal models (e.g. Zucker Diabetic Fatty (ZDF) rat [12], streptozotocin (STZ)-induced rat [13]); however, elevation of GK is reported in other diabetic animals [14-16]. Although how the difference is caused has not been clarified, it may be supposed that suppressed insulin is always accompanied by suppressed GK level.

Glycogen synthase (GS) and glycogen phosphorylase (GP) are rate-limited key enzymes of glycogen metabolism $[17,18]$. Glycogen content was decreased in pre-diabetic SDT rats, and the decrease was more pronounced at older age [4]. A decreased GS activity and a moderate decrease in glycogen store preceded development of diabetes in SDT rat. In diabetic state, GP activity and mRNA expression in liver were also decreased in SDT rats. GP activity is considered to be decreased as a consequence of elevation of serum glucose 
A

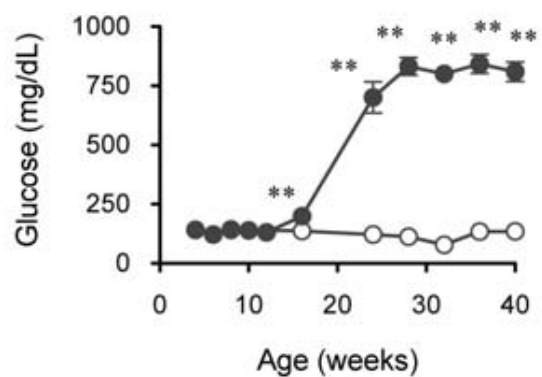

B

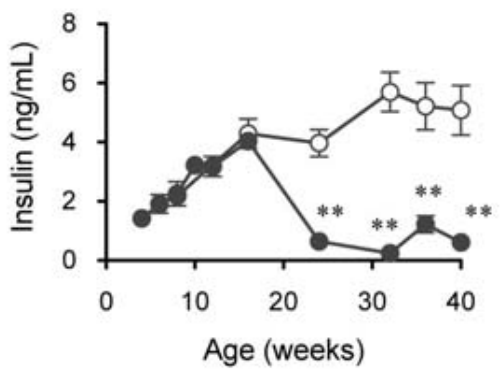

C

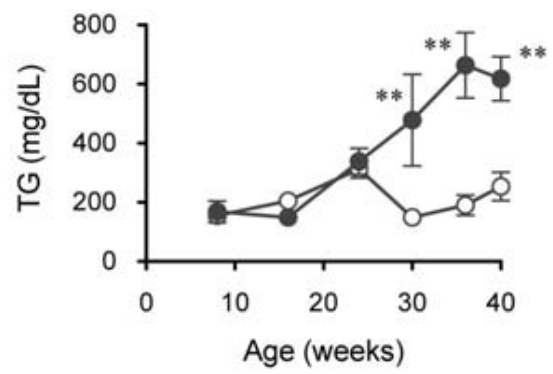

Fig. (1). Serial changes of biochemical parameters. (A) blood glucose, (B) insulin, and (C) TG levels in SD rats (open circles) and SDT rats (closed circles). Data represent means \pm S.E.M. $(\mathrm{n}=6-8) .{ }^{* *} \mathrm{p}<0.01$ ( $v s$ age-matched SD rat, unpaired $t$-test).

A

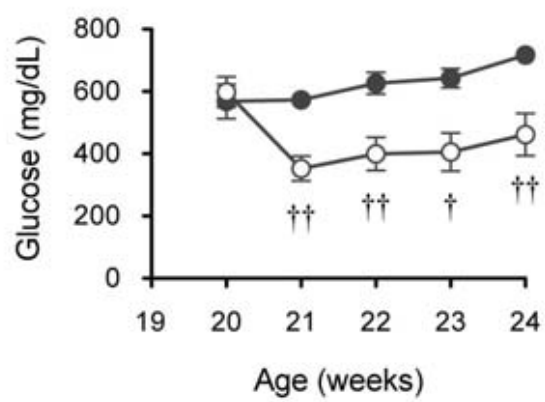

D

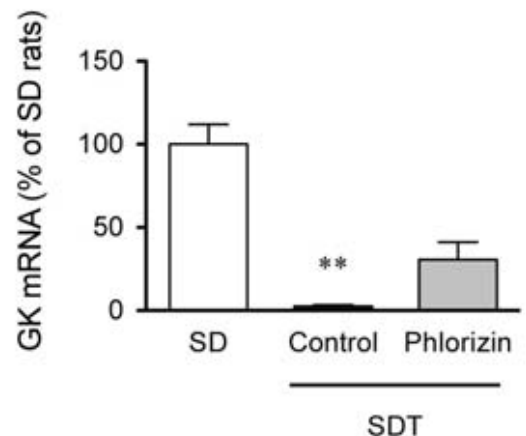

B

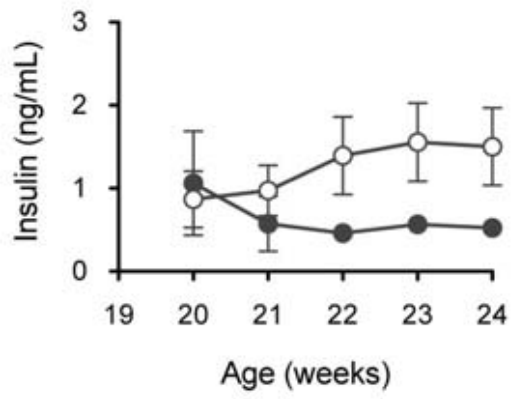

E

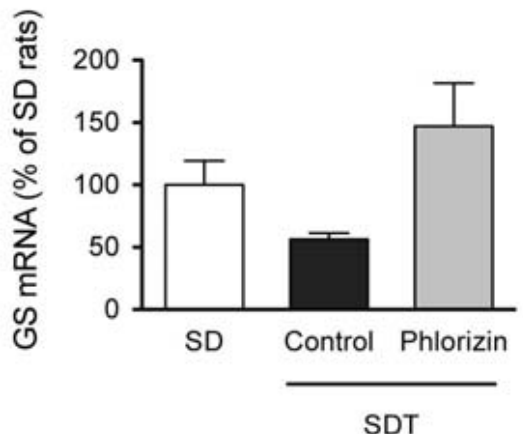

C

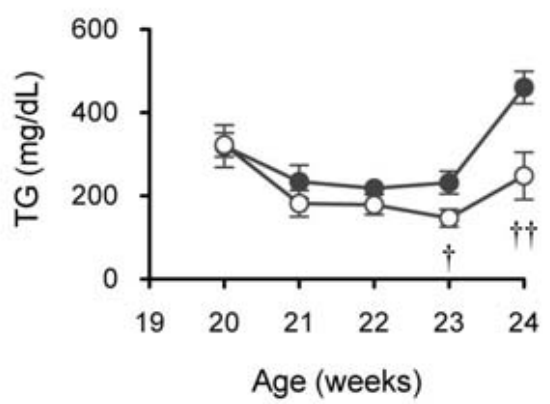

$\mathbf{F}$

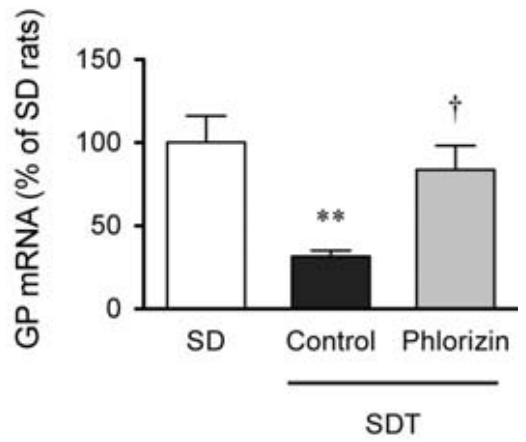

Fig. (2). Effect of phlorizin on blood biochemical parameters and key enzymes mRNA expression of hepatic glucose metabolism. (A) Blood glucose, (B) insulin, and (C) TG levels in control SDT rats (closed circles) and phlorizin-treated SDT rats (100 mg/kg, b.i.d, s.c.; open circles). Relative hepatic mRNA expressions of (D) GK, (E) GS, and (F) GP after 4 weeks treatment of phlorizin (24 weeks of age). Data represent means \pm S.E.M. $(\mathrm{n}=5-6)$. ${ }^{* *} \mathrm{p}<0.01$ ( $v s$ age-matched SD rat, unpaired $t$-test). $\uparrow \mathrm{p}<0.05$ ( $v s$ control SDT rat, unpaired $t$-test).

and reduction of glycogen stock. GP is known to exert high activity in response to attenuated insulin signal due to insulin deficiency, and low expression or activity in association with an increase in blood glucose, a decrease in glycogen store, an enhancement of gluconeogenesis [17], and an increase in glucagon/insulin ratio [19]. The glycogen metabolism pathway in diabetic SDT rats is broken down, thus glycogen can not play a role in glucose supply [4]. At least an increase in blood glucose and a decrease in glycogen store are considered to be applicable to the SDT model. Insulin treatment ameliorates hyperglycemia and prevents diabetic complications in SDT rats [20-23]. However, exogenous insulin itself has affected glucose metabolism in liver, and thus a drug that directly decreases blood glucose maybe useful to study effects of hypergycemia in metabolism. Phlorizin, a specific inhibitor of sodium-coupled glucose transporter (SGLTs), is reported to cause excretion of glucose into urine and lower blood glucose levels in several diabetic animal models $[24,25]$. SDT rats treated with phlorizin for 4 weeks from 20 weeks of age showed 
decreased blood glucose and TG, and insulin level was maintained (Fig. 2A-C). As result of phlorizin treatment, down-regulated expression of hepatic GK, GS, and GP mRNAs were recovered (Fig. 2D-F). These results support the idea that the hyperglycemia directly controls expression of hepatic enzymes responsible for glycogen metabolism.

Rate-limited enzymes of gluconeogenesis, such as glucose6-phosphate (G6Pase), fructose-1, 6-bisphosphatase (FBPase), and phosphoenolpyruvate carboxykinase (PEPCK) [26-28], also play important roles in glucose metabolism. Expression of these mRNAs was increased in diabetic, but not in pre-diabetic SDT rats [4]; hence the key enzymes of gluconeogenesis are upregulated in SDT rats after progression of diabetes.

\section{LIPID METABOLISM}

Decreased signals of insulin and leptin cause diabetic hyperphagia [29], and explains for hypertriglyceridemia in diabetes. In diabetic state, hyperphagia leads to intestinal hypertrophy [30], which contributes to increased cholesterol synthesis and lipid absorption. Therefore, the impaired chylomicron catabolism is closely associated with diabetic hypertriglyceridemia. In addition, overproduction of the TGrich lipoprotein may contribute to diabetic hypertriglyceridemia. Earlier studies indicated that increased TG absorption from the small intestine contributes to hypertriglyceridemia in diabetes [31-33].

SDT rat shows marked hypertriglyceridemia concomitant with hyperglycemia/hypoinsulinemia [6,7] (Fig. 1C). The major increase of TG in the circulation of SDT rats occurs in the TG-rich lipoprotein fraction chylomicron [5]. Lower plasma leptin level followed by hyperphagia [5] seems to cause intestinal hypertrophy in SDT rats [34] (Fig. 3). The small intestine plays a predominant role in the absorption of dietary lipids. Abnormal expression and/or activity of enzymes involved in intestinal TG synthesis and chylomicron assembling such as acyl-CoA:monoacylglycerol acyltransferase 2 (MGAT2), acyl-CoA:diacylglycerol acyltransferase 1 (DGAT1), and microsomal triglyceride transfer protein (MTP) have been observed in some in vitro and in vivo models of diabetes [3537]. SDT rats have increased intestinal mRNA of these enzymes and transfer protein [5] in addition to intestinal hypertrophy. Therefore, it is conceivable that increased intestinal mRNAs of these enzymes and transfer protein related to postprandial TG absorption are caused by extreme hypoinsulinemia/hyperglycemia.

Plasma TG and chylomicron levels are increased in diabetic animal models [38-41]. After olive oil loading, SDT rats showed higher plasma TG level compared to that of normal rats [5]. SDT rats also showed increased TG in lymph chylomicron and plasma TG accumulation after oil loading in diabetic state, indicating that postprandial TG absorption from intestine is increased in SDT rats in diabetic state (Fig. 4A, B). Interestingly, sustained plasma TG level elevation after oil loading was also observed in SDT rats before hyperglycemia/hypertriglyceridemia. In contrast, TG in lymph chylomicron and plasma TG accumulation after oil loading was not different from those of SD rats at this age (Fig. 4A, B). Significant delay in TG clearance was observed from 8 weeks of age (Fig. 5A, B). These results clearly indicate impaired TG catabolism of SDT rats from prediabetic stage and excess TG absorption at diabetic stage [5]. In a model of impaired insulin secretion (such as STZinduced diabetic rats), the high TG levels are probably due to low TG clearance because of reduced adipose tissue lipoprotein lipase (LPL) activity [42]. Lower LPL expression affects impaired TG catabolism in SDT rats (Fig. 5C). Diabetic SDT rats showed reduced hepatic TG secretion (Fig. 4C) as same as STZ rats. Plasma insulin level in STZ rats was negatively correlated with lipids and apoB in plasma and TG-rich lipoprotein [43]. Also, Mason et al. reported that decreased TGSR in STZ rats was improved by insulin treatment [44]. Therefore, the hypoinsulinemic environment may cause decreased TGSR in SDT rats. This characteristic on TGSR is different from hypertriglyceridemic model with insulin resistance and hyperglycemia that shows increased VLDL-TG. Hepatocytes from obese Zucker rats displayed higher total VLDL-TG secretion [45] and acute glucose infusion elevated plasma TG concentration [46].

\section{CONCLUSION}

Our previous study provided evidence for involvement of hepatic glucose metabolic disorder in onset and progress of diabetes in SDT rat [4]. As it is increasingly important to elucidate the mechanism of onset of diabetes and to develop drugs to prevent diabetic pathological progress, SDT rat should offer a highly useful model of spontaneous diabetic onset for such studies. In addition, abnormality in $\mathrm{TG}$ absorption and impaired lipid catabolism antecedent to hypoinsulinemia/hyperglycemia seem to cause postprandial hypertriglyceridemia in SDT rat [5]; hence, the characteristics of lipid metabolism in SDT rat can be useful in studies of diabetic hypertriglyceridemia and TG metabolism. SDT rats show impaired glucose tolerance and insulin response to oral glucose loading in pre-diabetic state [8]. The relation-
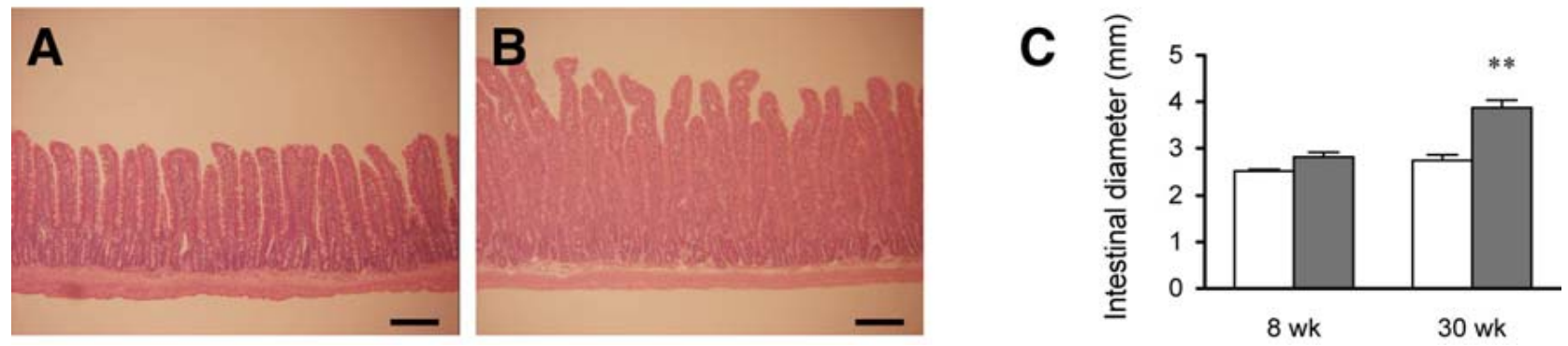

Fig. (3). Histopathological changes of small intestine. Typical microphotographs of jejunum from (A) SD rat and (B) SDT rat at 30 weeks of age (HE stain; bar $=400 \mu \mathrm{m})$. (C) Diameter of jejunum $(\mathrm{n}=6)$. Open Column: SD rat, closed column: SDT rat. ** $<<0.01$ ( $v s$ age-matched SD rat, unpaired $t$-test). 
A

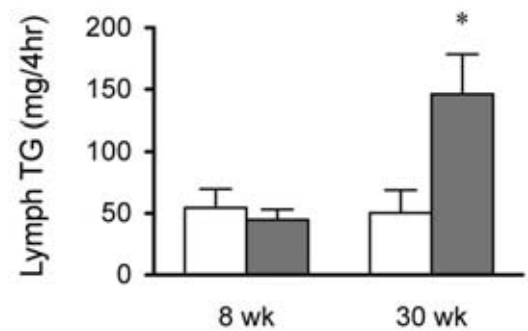

B

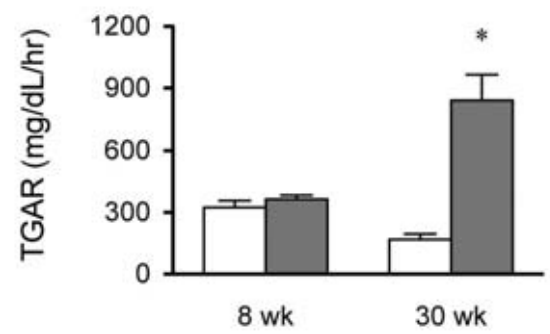

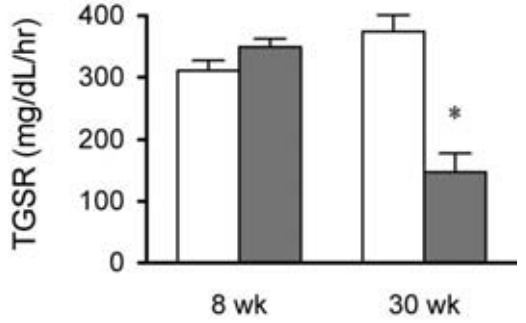

Fig. (4). Intestinal fat absorption and hepatic TG secretion. (A) Mesenteric lymph chylomicron collected for $4 \mathrm{~h}$ after olive oil loading. (B) Plasma TG absorption rate (TGAR) after olive oil and Triton WR1339 administration. (C) Hepatic TG secretion rate (TGSR) after Triton WR1339 injection. Open Column: SD rat, closed column: SDT rat. Data represent means \pm S.E.M. $(\mathrm{n}=3-6) .{ }^{*} \mathrm{p}<0.05$, $(v s$ age-matched SD rat, unpaired $t$-test). Figures modified from [5].

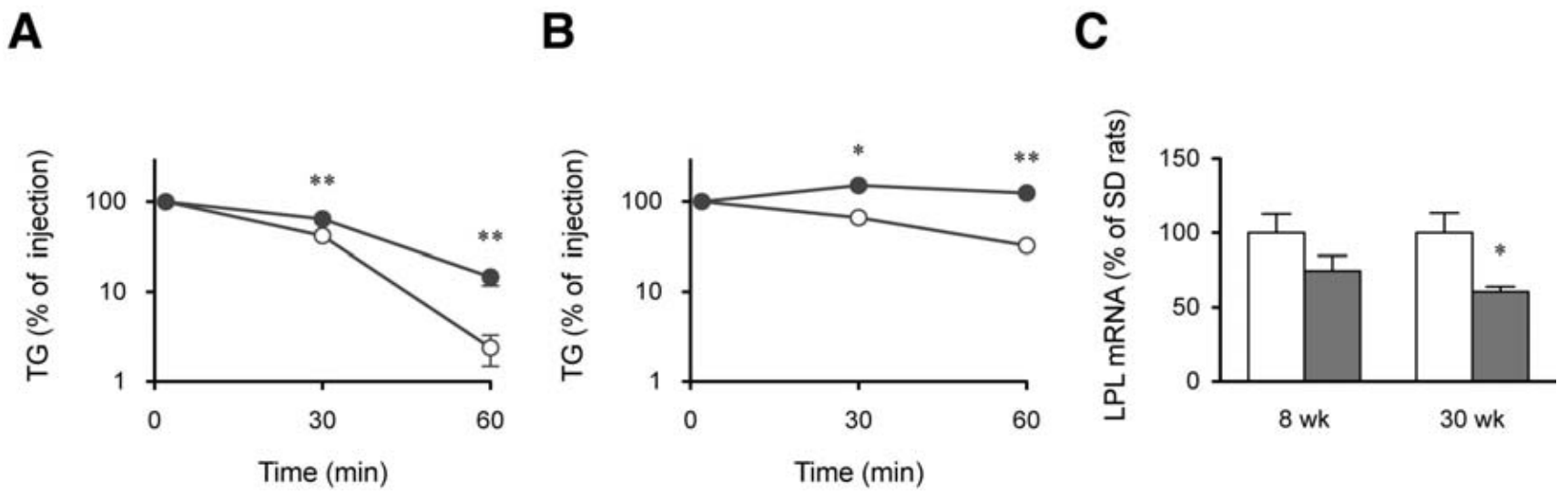

Fig. (5). TG clearance from circulation in SD rats (open circles) and SDT rats (closed circles) at (A) 8 weeks and (B) 30 weeks of age. (C) Epididymal fat lipoprotein lipase (LPL) mRNA expression in SD rats (open column) and SDT rats (closed column). Data represent means \pm S.E.M. $(\mathrm{n}=6-11) .{ }^{*} \mathrm{p}<0.05, * * \mathrm{p}<0.01$ ( $v s$ age-matched SD rat, unpaired t-test). Figures modified from [5].

ship between impaired lipid catabolism and impaired glucose tolerance in the pre-diabetic state also seems to offer some keys towards clarification of the onset mechanism of diabetes.

\section{REFERENCES}

[1] Howard BV. Lipoprotein metabolism in diabetes mellitus. J Lipid Res 1987; 28: 613-28.

[2] Garcia MJ, McNamara PM, Gordon T, Kannel WB. Morbidity and mortality in diabetics in the Framingham population. Sixteen year follow-up study. Diabetes 1974; 23: 105-11.

[3] Steiner G. Diabetes and atherosclerosis: an overview. Diabetes 1981; 30: 1-7.

[4] Morinaga H, Yamamoto H, Sakata K, et al. Characterization of hepatic glucose metabolism disorder with the progress of diabetes in male Spontaneously Diabetic Torii rats. J Vet Med Sci 2008; 70: 1239-45.

[5] Sasase T, Morinaga H, Yamamoto H, et al. Increased fat absorption and impaired fat clearance cause postprandial hypertriglyceridemia in Spontaneously Diabetic Torii rat. Diabetes Res Clin Pract 2007; 78: 8-15.

[6] Shinohara M, Masuyama T, Kakehashi A. The Spontaneously Diabetic Torii (SDT) rat with retinopathy lesions resembling those of humans. In: Shafrir E, Eds. Animal models of diabetes: frontiers in research. 2nd ed. Boca Raton (FL): CRC Press 2007; pp. 311-21.

[7] Shinohara M, Masuyama T, Shoda T, et al. A new spontaneously diabetic non-obese Torii rat strain with severe ocular complications. Int J Exp Diabetes Res 2000; 1: 89-100.

[8] Masuyama T, Komeda K, Hara A, et al. Chronological characterization of diabetes development in male Spontaneously Diabetic Torii rats. Biochem Biophys Res Commun 2004; 314: 870-7.
[9] Ferre T, Riu E, Bosch F, Valera A. Evidence from transgenic mice that glucokinase is rate limiting for glucose utilization in the liver. FASEB J 1996; 10: 1213-8.

[10] Basu A, Basu R, Shah P, et al. Type 2 diabetes impairs splanchnic uptake of glucose but does not alter intestinal glucose absorption during enteral glucose feeding: additional evidence for a defect in hepatic glucokinase activity. Diabetes 2001; 50: 1351-62.

[11] Basu A, Basu R, Shah P, et al. Effects of type 2 diabetes on the ability of insulin and glucose to regulate splanchnic and muscle glucose metabolism: evidence for a defect in hepatic glucokinase activity. Diabetes 2000; 49: 272-83.

[12] Munoz MC, Barbera A, Dominguez J, et al. Effects of tungstate, a new potential oral antidiabetic agent, in Zucker diabetic fatty rats. Diabetes 2001; 50: 131-8.

[13] Lavoie L, Dimitrakoudis D, Marette A, et al. Opposite effects of hyperglycemia and insulin deficiency on liver glycogen synthase phosphatase activity in the diabetic rat. Diabetes 1993; 42: 363-6.

[14] Aiston S, Peak M, Agius L. Impaired glycogen synthesis in hepatocytes from Zucker fatty fa/fa rats: the role of increased phosphorylase activity. Diabetologia 2000; 43: 589-97.

[15] Toyoda Y, Ito Y, Tanigawa K, Miwa I. Impairment of glucokinase translocation in cultured hepatocytes from OLETF and GK rats, animal models of type 2 diabetes. Arch Histol Cytol 2000; 63: 2438.

[16] Yen TT, Stamm NB. Constitutive hepatic glucokinase activity in $\mathrm{db} / \mathrm{db}$ and ob/ob mice. Biochim Biophys Acta 1981; 657: 195-202.

[17] Bollen M, Keppens S, Stalmans W. Specific features of glycogen metabolism in the liver. Biochem J 1998; 336 (Pt 1): 19-31.

[18] Gomis RR, Ferrer JC, Guinovart JJ. Shared control of hepatic glycogen synthesis by glycogen synthase and glucokinase. Biochem J 2000; 351 Pt 3: 811-6. 
[19] Roesler WJ, Khandelwal RL. Regulation of rat liver glycogen phosphorylase concentration by in vivo relative levels of glucagon and insulin. Endocrinology 1987; 121:227-32.

[20] Ohta T, Matsui K, Miyajima K, et al. Effect of insulin therapy on renal changes in spontaneously diabetic Torii rats. Exp Anim 2007; 56: $355-62$.

[21] Sasase T, Morinaga H, Abe T, et al. Protein kinase $\mathrm{C}$ beta inhibitor prevents diabetic peripheral neuropathy, but not histopathological abnormalities of retina in Spontaneously Diabetic Torii rat. Diabetes Obes Metab 2009; 11: 1084-7.

[22] Sasase T, Ohta T, Ogawa N, et al. Preventive effects of glycaemic control on ocular complications of Spontaneously Diabetic Torii rat. Diabetes Obes Metab 2006; 8: 501-7.

[23] Sasase T, Ohta T, Ogawa N, et al. Diabetic complications of Spontaneously Diabetic Torii rat. Diabetes 2005; 54 (Suppl 1): A221.

[24] Blondel O, Bailbe D, Portha B. Insulin resistance in rats with noninsulin-dependent diabetes induced by neonatal (5 days) streptozotocin: evidence for reversal following phlorizin treatment. Metabolism 1990; 39: 787-93.

[25] Khan A, Efendic S. Evidence that increased glucose cycling in islets of diabetic ob/ob mice is a primary feature of the disease. Am J Physiol 1995; 269: E623-6.

[26] Barzilai N, Rossetti L. Role of glucokinase and glucose-6phosphatase in the acute and chronic regulation of hepatic glucose fluxes by insulin. J Biol Chem 1993; 268: 25019-25.

[27] Hanson RW, Patel YM. Phosphoenolpyruvate carboxykinase (GTP): the gene and the enzyme. Adv Enzymol Relat Areas Mol Biol 1994; 69: 203-81.

[28] Pilkis SJ, Granner DK. Molecular physiology of the regulation of hepatic gluconeogenesis and glycolysis. Annu Rev Physiol 1992; 54: 885-909.

[29] Havel PJ, Uriu-Hare JY, Liu T, et al. Marked and rapid decreases of circulating leptin in streptozotocin diabetic rats: reversal by insulin. Am J Physiol 1998; 274: R1482-91.

[30] Young NL, Saudek CD, Walters L, Lapeyrolerie J, Chang V. Preventing hyperphagia normalizes 3-hydroxy-3-methylglutarylCoA reductase activity in small intestine and liver of diabetic rats. $\mathrm{J}$ Lipid Res 1982; 23: 831-8.

[31] Popper DA, Shiau YF, Reed M. Role of small intestine in pathogenesis of hyperlipidemia in diabetic rats. Am J Physiol 1985; 249: G161-7.

[32] Risser TR, Reaven GM, Reaven EP. Intestinal very low density lipoprotein secretion in insulin-deficient rats. Diabetes 1978; 27: 902-8.
[33] Steiner G, Poapst M, Davidson JK. Production of chylomicron-like lipoproteins from endogenous lipid by the intestine and liver of diabetic dogs. Diabetes 1975; 24: 263-71.

[34] Yamada K, Hosokawa M, Fujimoto S, et al. The spontaneously diabetic Torii rat with gastroenteropathy. Diabetes Res Clin Pract 2007; 75: 127-34.

[35] Chen HC, Smith SJ, Ladha Z, et al. Increased insulin and leptin sensitivity in mice lacking acyl CoA:diacylglycerol acyltransferase 1. J Clin Invest 2002; 109: 1049-55.

[36] Meegalla RL, Billheimer JT, Cheng D. Concerted elevation of acyl-coenzyme A:diacylglycerol acyltransferase (DGAT) activity through independent stimulation of mRNA expression of DGAT1 and DGAT2 by carbohydrate and insulin. Biochem Biophys Res Commun 2002; 298: 317-23.

[37] Mostafa N, Bhat BG, Coleman RA. Increased hepatic monoacylglycerol acyltransferase activity in streptozotocin-induced diabetes: characterization and comparison with activities from adult and neonatal rat liver. Biochim Biophys Acta 1993; 1169: 189-95.

[38] Bar-On H, Roheim PS, Eder HA. Serum lipoproteins and apolipoproteins in rats with streptozotocin-induced diabetes. J Clin Invest 1976; 57: 714-21.

[39] Brown DF. Triglyceride metabolism in the alloxan-diabetic rat. Diabetes 1967; 16: 90-5.

[40] Reaven EP, Reaven GM. Mechanisms for development of diabetic hypertriglyceridemia in streptozotocin-treated rats. Effect of diet and duration of insulin deficiency. J Clin Invest 1974; 54: 1167-78.

[41] Staprans I, Pan XM, Rapp JH, Feingold KR. Chylomicron and chylomicron remnant metabolism in STZ-induced diabetic rats. Diabetes 1992; 41: 325-33.

[42] Redgrave TG, Callow MJ. The effect of insulin deficiency on the metabolism of lipid emulsion models of triacylglycerol-rich lipoproteins in rats. Metabolism 1990; 39: 1-10.

[43] Hirano T, Mamo JC, Takeuchi H, Nagano S, Takahashi T. Correlation of insulin deficiency and hypertriglyceridemia in diabetic rats. Diabetes Res Clin Pract 1991; 12: 173-80.

[44] Mason TM, Chan B, El-Bahrani B, et al. The effect of chronic insulin delivery via the intraperitoneal versus the subcutaneous route on hepatic triglyceride secretion rate in streptozotocin diabetic rats. Atherosclerosis 2002; 161: 345-52.

[45] Kalopissis AD, Griffaton G, Fau D. Inhibition of hepatic very-lowdensity lipoprotein secretion in obese Zucker rats adapted to a highprotein diet. Metabolism 1995; 44: 19-29.

[46] Hirano T, Mamo JC, Furukawa S, Nagano S, Takahashi T. Effect of acute hyperglycemia on plasma triglyceride concentration and triglyceride secretion rate in non-fasted rats. Diabetes Res Clin Pract 1990; 9: 231-8. 